\title{
Utilizing Low-Dose Transmission Electron Microscopy for Structure and Defect Identification in Group III - Nitride Electronic Devices
}

Petra Specht ${ }^{1}$, Martina Luysberg ${ }^{2}$, J. Chavez ${ }^{1}$, T.R. Weatherford ${ }^{3}$, T.J. Anderson ${ }^{4}$, A.D. Koehler ${ }^{4}$, C. Kisielowski ${ }^{5}$

1. University of California, Berkeley, Dept. of Materials Science \& Engineering, Berkeley, CA, USA

2. Ernst Ruska Centre, Research Centre Juelich, Juelich, Germany

3. Naval Postgraduate School, Monterey, CA, USA

4. Naval Research Laboratory, Washington DC, USA

5. Lawrence Berkeley National Laboratory, Molecular Foundry, Berkeley, CA, USA

Group III - nitride materials are already used in multiple high speed transistor applications as well as in opto-electronics, from light emitting diodes to photodiodes in sensors. Recently, bulk like GaN became available which also enables the development of vertical power device structures due to significantly reduced dislocation density (approximately from $10^{8} / \mathrm{cm}^{2}$ to $10^{4} / \mathrm{cm}^{2}$ ). Device optimization for any such device structures requires imaging techniques which maintain original, often highly defective device areas, at heterostructure interfaces, in the vicinity of metal contacts or otherwise locally strained, selected material areas, such as in locally dopant implanted regions.

For these, often highly complex material systems TEM sample preparation which maintains the areas of interest in their pristine condition is demanding. The technique of choice is usually a focused ion beam cross section followed by a low voltage Ar ion mill (Nanomill, Fischione), to carefully thin and clean the FIB-induced damaged surface areas. Here, imaging is performed in low dose HR-TEM, utilizing a Nelsonian illumination scheme [1], in an aberration-corrected microscope with monochromated electron beam (TEAM, FEI).

As an example, we investigated $\mathrm{Mg}$-implanted $\mathrm{GaN}$ regions which are needed to locally increase p-doping concentrations in $\mathrm{GaN}$, to improve a p-type contact for a vertical power switch. Magnesium implantation, however, locally increases damage in the affected $\mathrm{GaN}$ area which is only partially recovered in a novel, 2 step, multi-cycle annealing procedure. In order to optimize this process, one needs to determine which defects are formed at which process step, and how those defects will affect the overall device performance, here the p-type conductivity.

Using dose rates between 30 and $300 \mathrm{e}^{-} / \AA^{2}$ s image series with varying foci were obtained and exit wave reconstructions calculated. The phase images of the exit waves show after the second annealing step a number of extended defects, including bubbles, likely filled with nitrogen, dislocation loops and small $\mathrm{MgO}$ particles. Figure 1 displays those images. A "super-structure" in the GaN lattice was observed which forms under the electron beam in both, single and double annealed Mg-implanted GaN. The aforementioned "bubbles" move under the electron beam, the image shown in Figure 1 therefore was shot "in the dark", with only $30 \mathrm{e}^{-} / \AA^{2}$ s dose rate which kept the defect in place. If one wants to obtain original, not e-beam induced defect structures, the use of low electron doses is mandatory in such material structures [2]. 
References:

[1] C. Kisielowski et al, Micron 68 (2015), p. 186.

[2] Work at the Molecular Foundry was supported by the Office of Science, Office of Basic Energy Sciences, of the U.S. Department of Energy under Contract No. DE-AC02-05CH11231.

Figure 1. Mg implanted GaN quasi-bulk substrate, double annealed in a multi-cycle RTA mode. Left, bottom: phase image of an exit wave (approx. $100 \mathrm{e}^{-} / \AA^{2} \mathrm{~s}$ ) with an e-beam induced varying dark/bright "super-structure", a MgO nano-cluster at the edge of the TEM sample and a dislocation loop (white square) depicted. Right: phase image of the same material showing a nitrogen bubble, approx. $3 \mathrm{~nm}$ wide, electron dose for this image series was approx. $30 \mathrm{e}^{-} / \AA^{2}$ s. Insets show the corresponding FFTs, also some areas are magnified for better visibility. Top, middle shows one of the typical dislocation loops of this sample as it is imaged in conventional contrast imaging.

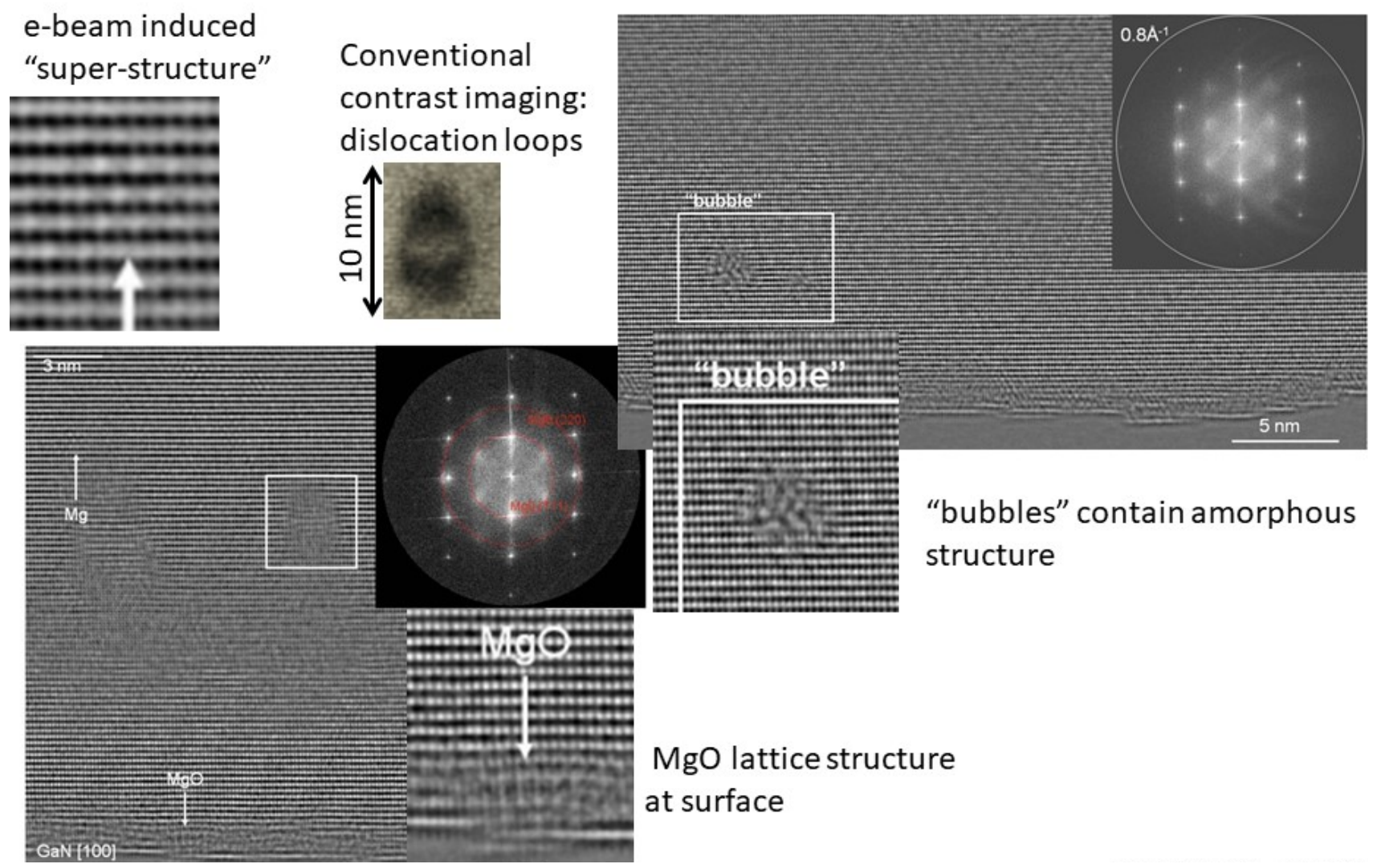

